\title{
Psychology and Psychoanalysis
}

RESEARCH ARTICLE

\section{Factor Structure and Psychometric Properties of Cognitive- Behavioral Scales in Caregivers of Persons with Dementia}

\author{
Abir K Bekhet ${ }^{*}$ and Mauricio Garnier-Villarreal
}

Marquette University College of Nursing, USA

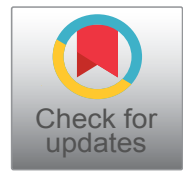

*Corresponding author: Abir K Bekhet, PhD, RN, HSMI, Department of Mental Health Nursing, Marquette University College of Nursing, Clark Hall 530 N, 16th Street, Milwaukee, WI 53233, USA, Tel: 414-288-3847

\begin{abstract}
Background/Purpose: Caregiving can be costly to dementia caregivers' well-being. Assessing the factor structure and psychometric properties of Cognitive-Behavioral Scales in dementia caregivers is an essential step in addressing the gap in the current state of research. Specifically, it is essential to determine first whether the factorial structure of the three measures used in this study namely, the Positive Thinking Skills Scale, the Revised Memory and Behavior Problems Checklist, and the Zarit Burden Interview are good representation of the data by studying the good model fit. Next, evaluating the reliability of each factor of the three measures used are essential to learn about the precision of the factors. Lastly, it is vital to study the factor correlation and its relevance to the theory used to determine the validity of the factors.
\end{abstract}

Methods: A descriptive, correlational, cross-sectional design in a convenience sample of 100 caregivers.

Results: Results indicated that the factorial structure of the three scales is a good representation of the data; an acceptable reliability of each factor of the three measures; and the factors correlated as expected and showed their relevance to the underlying theory.

Conclusions: Future studies might consider studying the mediating/moderating effects of positive thinking on carerecipients challenging behavior problems. The findings can be used as a guide to provide a positive thinking training intervention among caregivers.

\section{Keywords}

Factor structure, Psychometric properties, The Zarit Burden Interview, Positive thinking skills scale, The revised memory and behavior problems checklist, Dementia caregivers

\section{Factor Structure and Psychometric Properties of Cognitive-Behavioral Scales in Caregivers of Persons with Dementia}

By the year 2050, there is a projection that the number of American people diagnosed with dementia will raise to 16 million; triple the current number of 5.4 million [1]. Dementia is characterized by declines in cognitive functions and eventually impairing the individual's abilities to carry out daily activities $[2,3]$. Eighty-percent of the care needed is provided at home by family members who assume the role of informal care giving [4]. Caregiving can be costly to caregivers' physical and psychological health. Dementia caregivers report poor self-rated health, anxiety, anger, fatigue, and poor quality of life $[3,5]$. Also, caregivers describe feelings of confusion, uncertainty, sense of isolation, and disbelief [6-8]. Sense of loss, fear, and anger have also been reported by caregivers [6,9]. In addition, previous research has shown that almost half of dementia caregivers meet the diagnostic criteria for clinical depression and the spouses of persons with dementia are four time more liable to develop depression as compared to spouses of persons without dementia $[2,10,11]$.

Yet some dementia caregivers develop resilience and report that caregiving results in an enhanced sense of meaning in life and feelings of joy $[12,13]$. These people, were clearly able to rise above their challenges. Addressing the gap in the current state of research with family caregivers of persons with dementia is essential. Specifically, targeting caregivers' positive 
thinking, a protective factor that may buffer the effects of chronic burden on their care recipients' challenging behaviors is imperative. However, it is essential to determine first whether the factorial structure of the three measures used in this study namely, the Positive Thinking Skills Scale (PTSS), the Revised Memory and Behavior Problems Checklist (RMBPC), and the Zarit Burden Interview (ZBI) are good representation of the data by studying the good model fit. Next, evaluating the reliability of each factor of the three measures used are essential to learn about the precision of the factors. Lastly, it is vital to study the factor correlation and its relevance to the theory used to determine the validity of the factors.

\section{Background on Factor Structure of the Measures Used}

Persons with dementia exhibit behavioral complications such as emotional disturbances and disruptive behaviors, which can be challenging and can impact the caregivers' physical and psychological well-being [14]. Standardized instruments that assess the occurrence and severity of persons with dementia behavioral problems are essential not only for assessing persons with dementia behavioral disturbance but also vital in identifying caregivers' challenges [14].

The Revised Memory and Behavior Problems Checklist (RMBPC) provides assessments of the frequency of patients' behavioral problems [15]. Exploratory factor analyses of the RMBPC showed multiple dimensions underlie this measure. However, previous research showed significant inconsistencies not only in item content but also the resulting factor structures, which ranged from three to eight [15-17]. In addition, measuring the needs and the burden of the family caregivers are essential for assessment and intervention. Therefore, a validated measure that assess the burden and challenges can't be overlooked. In fact, previous research lacks details regarding factorial analysis and psychometric information. For example, the research conducted by Fuh and colleagues [17] did not test the factorial structure of the checklist and limited their analysis to test-retest comparison between sum scores. Other researchers such as Dura and colleagues; Roth and colleagues [14,16] and Teri and colleagues [15] did a Principal Component Analysis (PCA) and interpreted it as a Factor Analysis (FA). Given the fact that PCA cannot identify underlying meaningful factors (like FA do) any interpretation from these are invalid $[18,19]$. PCA and FA are clearly different methods, with different objectives, PCA is commonly (mistakenly) use for Factor Analysis. This leads to a gap in proper Factor Analysis for the test of the underlying factors [18,19]. Allen and Colleagues tested the factorial structure with a Confirmatory Factor Analysis and compared treating the items as continuous and as ordered-categorical. Results indicated that when the items were treated as ordered-categorical, there was an improvement in model fit. This, in fact, is an indication that treating the Likert type items as continuous represents a model misspecification.

The Zarit Burden Interview (ZBI) is a widely used measure that assesses the caregivers perceived burden. Although previous research has examined the factor structure of the ZBI, inconsistencies have been reported [15]. Namely, two factor structures, three, and five have been identified in previous research [20-24]. Lai [20] based the Confirmatory Factor Analysis (CFA) in a preliminary exploratory PCA interpreted as Exploratory Factor Analysis (EFA). On the other hand, Longmire and Knight; Knight and collages [22,25] performed a proper Confirmatory Factor Analysis while treating the items as continuous which can be seen as a model misspecification with Likert type items, instead of treating them as ordered-categorical items.

Lastly, it is important to study the factorial structure of the Positive Thinking Skills Scale (PTSS), given the fact it is a new scale and has been used in limited study. Therefore, the purpose of this study is:

1. To determine which factorial structure for the three measures used in this study namely, PTSS, RMBPC, and the $\mathrm{ZBI}$, are good representation of the data by studying the model fit and the theoretical relevance.

2. To evaluate the reliability of each factor of the three measures used to assess the precision of the selected factor structure.

3. To determine the factor correlation and its relevance to the theory used.

\section{Methodology}

\section{Research design}

A descriptive, correlational, cross-sectional design was used in this study.

\section{Subjects/setting}

A convenience sample of 100 caregivers of persons with dementia (CPWD), both men and women, of all ages and living arrangements comprised the study sample.

\section{Procedures for data collection}

Prior to recruitment of CPWD, approval was obtained from the Institutional Review Board (IRB). The researcher contacted the administrators at the Alzheimer's Association early stage programs in Southeastern Wisconsin to explain the purpose of the study and the data collection procedures and to gain their help in recruiting CPWD. After obtaining administrative approval, notices describing the research and personal contact was posted in Southeastern Wisconsin by the administrators. Also, CPWD were recruited from "Walk to End Alzheimer". Those who are interested contacted 
the research assistant. On an agreed upon date and time, the research assistant met with the subjects in which she explained the purpose of the study and she asked them to complete the consent form and then the study questionnaires data. Questionnaires were distributed to caregivers and the research assistant doubled checked the completion of each questionnaire in order to minimize missing data.

\section{Measures}

Caregiver burden was measured by the Zarit Burden Interview (ZBI) [22]. The $\mathrm{ZBI}$ is composed of 22 items that indicate the burden caregivers sometimes feel when they are taking care of their relatives. Items are scored on a 5-point Likert scale ranging from never (0) to nearly always (4). In previous research, Cronbach's alphas for the ZBI range ranged 0.88-0.92 [22,26,27].

Positive Thinking Skills Scale (PTSS) is an eight-item scale that measures positive thinking as a direct measure of intervention fidelity [28]. All items are scored in the positive direction; higher scores indicate more positive thinking. Response options are four-point Likert scales ranging from $0=$ never to 3 = always. In a study with caregivers of persons with autism spectrum disorders, Bekhet and Zauszniewski reported Chronbach's alpha of 0.90 . Construct validity was supported by correlations in the expected directions with measures of positive cognitions $(r=0.53 ; p<0.01)$, resourcefulness $(r=0.63$; $p<0.01)$, depression $(r=-0.45 ; p<0.01)$ and general well-being $(r=0.40 ; p<0.01)$ for the Positive Thinking Skills Scale (PTSS) [28].

The Revised Memory and Behavior Problems Checklist (RMBPC) is the subscale on the frequency of a person with dementia behavior $[14,29]$. The RMBPC is a 24-item scale that assesses the occurrence of persons with dementia behaviors using an objective scaling criterion. Behaviors are rated by caregivers from never occurs (0) to occurs daily or more often (4). Chronbach's alpha reported in previous study was $\alpha(0.89)[14,29]$.

\section{Data analysis}

The data analysis was done in R [30]. The data analysis approach was using Structural Equation Modeling (SEM), with the R package lavaan [31]. This framework allows us to reduce measurement error of the instruments and to estimate a more precise measure of the latent factor underlying the scale items [32,33]. As presented by Raykov [34], the SEM framework presents beneficial conditions to develop and test scales, such as evaluation of multidimensional structures, correlations between constructs, evaluation of multiple reliability measures, and correction for measurement error.

Missing data was handled with Multiple Imputation (MI), a modern method to properly handle missing data, improving parameter recoverability, reducing bias, and increasing power [35-37]. MI was done with the R package mice [38], and the imputations were analyzed with the semTools package [39].

Reliability was evaluated with multiple indices, including the most common estimate of reliability is the Cronbach's coefficient alpha $(\alpha, 1951)$ [40]. This has shown disadvantages in underestimating or overestimating, which makes it a mis-estimator. This is due to how $\alpha$ approximates reliability in function of inter item correlation $[34,41]$. Due to the known limitations of $\alpha$, we present two improved estimates of reliability. First, hierarchical $\omega$ [42] which is a conservative estimate; and second the Maximal Reliability (MR) coefficient. MR estimates the reliability of a scale assuming items have a different weight into it. MR is the maximal possible reliability for a linear combination of the scale items. MR involves the estimation of the Optimal Linear Combination (OLC), which are the weights for each item $[34,43]$. Lastly, these estimates of construct reliability are presented with their respective Average Variance Extracted (AVE) [44]. This is a measure of the amount of variance that is capture from the items by the construct in relation to the amount of variance due to measurement error. MR, $\alpha, \omega$, and AVE are estimated with the R package semTools [39].

All the items from scales are answered in an ordered Likert scale. Given this, we are treating the items as ordered categorical instead of continuous, treating them as continuous would represent a misspecification of the model. We follow the categorical Confirmatory Factor Analysis (CFA) approach that analyses the data in function of the polychoric correlation between ordered items. This correlation assumes that there is an unobserved underlining variable that accounts for the ordered response [45]. For the CFA, data were analyzed with the Diagonal Weighted Least Square (DWLS) estimator, with mean and variance adjusted standard errors and chi-square statistic; this approach has shown to present reliable parameter estimates and model fit without the requirement of extremely large samples [45].

\section{Results}

The initial theoretical factor structure is as follows, positive thinking scale is represented by one factor, burden scale is represented by three factors (embarrassment/anger, patient's dependency, and selfcriticism), and finally the revised memory and behavior problem checklist is represented by three factors (memory, depression, and disruption). This factor structure reflects the expected subscales from each one $[22,28,29]$. This indicates that the PTSS measures one overall underlying factor representing positive thinking. On the other hand, the ZBI measures three dimensions of burden represented by embarrassment/anger, patient's dependency, and self-criticism. Lastly, the RMBPC measures three dimensions namely: memory, depression, and disruption. 
The measurement CFA presents good fit $(X 2(969)=$ 1054.803, $p=0.028$, RMSEA $=0.030[90 \% \mathrm{Cl}=0.011$, 0.042 , gamma-hat $=0.963$, adjusted gamma-hat $=$ 0.959), showing good absolute fit and indicating that the factorial structure is a good representation of the data $[32,33]$. As CFA is a generative process, this factorial structure is a likely representation of the data generation process for the participants responses.

Even as this model presented good model fit, we compared this structure to a factorial structure where the burden scale is represented by one factor instead of three. Testing these nested models, we find that these models cannot be assumed to represent the data equally $(\Delta X 2(3)=168.85, p<0.001)$, which tells that the three-factor structure is a better representation of the data. The factorial structure for the revised memory and behavior problem checklist was tested as well, again testing the original three factor structure against a one factor model, when testing these nested models, we find that we cannot assume both structures represent the data equally $(\Delta \times 2(3)=316.575, p<0.001)$. Meaning the three-factor structure is a better representation of the data.

Once the factorial structure has been established, we evaluate the reliability for each factor. Table 1 presents the reliability estimates for each factor, based

Table 1: Factor reliability measures for the selected factorial structure.

\begin{tabular}{|l|l|l|l|l|}
\hline & MR & $\boldsymbol{\alpha}$ & $\boldsymbol{\omega}$ & AVE \\
\hline Embarrassment/Anger & 0.831 & 0.806 & 0.805 & 0.370 \\
\hline Reaction to patient's dependency & 0.836 & 0.814 & 0.783 & 0.544 \\
\hline Self-criticism & 0.853 & 0.889 & 0.853 & 0.800 \\
\hline Positive thinking & 0.909 & 0.919 & 0.893 & 0.601 \\
\hline Memory related problems & 0.944 & 0.929 & 0.938 & 0.681 \\
\hline Depression & 0.968 & 0.927 & 0.939 & 0.619 \\
\hline Disruption & 0.875 & 0.866 & 0.862 & 0.471 \\
\hline
\end{tabular}

Table 2: Factor loadings and $\mathrm{R}^{2}$ for the selected factor structure.

\begin{tabular}{|c|c|c|c|c|}
\hline Factor/item $\#$ & Estimate (SE) & p-value & $95 \% \mathrm{Cl}$ & $\mathbf{R}^{2}$ \\
\hline \multicolumn{5}{|c|}{ Embarrassment } \\
\hline ZBI4 & $0.443(0.086)$ & $<0.001$ & $0.275,0.611$ & 0.196 \\
\hline ZBI5 & $0.373(0.088)$ & $<0.001$ & $0.201,0.546$ & 0.139 \\
\hline ZBI6 & $0.602(0.076)$ & $<0.001$ & $0.453,0.751$ & 0.362 \\
\hline ZBI9 & $0.713(0.061)$ & $<0.001$ & $0.593,0.833$ & 0.509 \\
\hline ZBI10 & $0.779(0.057)$ & $<0.001$ & $0.668,0.890$ & 0.607 \\
\hline ZBI11 & $0.633(0.065)$ & $<0.001$ & $0.506,0.760$ & 0.401 \\
\hline ZBI13 & $0.568(0.082)$ & $<0.001$ & $0.408,0.729$ & 0.323 \\
\hline ZBI18 & $0.656(0.067)$ & $<0.001$ & $0.525,0.786$ & 0.430 \\
\hline \multicolumn{5}{|c|}{ Reaction to patient dependency } \\
\hline ZBI2 & $0.814(0.050)$ & $<0.001$ & $0.715,0.913$ & 0.662 \\
\hline ZBI8 & $0.533(0.078)$ & $<0.001$ & $0.380,0.686$ & 0.284 \\
\hline ZBI12 & $0.890(0.044)$ & $<0.001$ & $0.804,0.976$ & 0.793 \\
\hline ZBI14 & $0.655(0.073)$ & $<0.001$ & $0.511,0.799$ & 0.429 \\
\hline \multicolumn{5}{|l|}{ Self-criticism } \\
\hline ZBI20 & $0.894(0.019)$ & $<0.001$ & $0.857,0.932$ & 0.800 \\
\hline ZBI21 & $0.894(0.019)$ & $<0.001$ & $0.857,0.932$ & 0.800 \\
\hline \multicolumn{5}{|c|}{ Positive Thinking } \\
\hline pt1 & $0.831(0.038)$ & $<0.001$ & $0.757,0.905$ & 0.691 \\
\hline pt2 & $0.816(0.053)$ & $<0.001$ & $0.712,0.920$ & 0.666 \\
\hline pt3 & $0.648(0.049)$ & $<0.001$ & $0.552,0.744$ & 0.420 \\
\hline pt4 & $0.574(0.064)$ & $<0.001$ & $0.447,0.700$ & 0.329 \\
\hline pt5 & $0.754(0.050)$ & $<0.001$ & $0.657,0.852$ & 0.569 \\
\hline pt6 & $0.883(0.035)$ & $<0.001$ & $0.814,0.952$ & 0.779 \\
\hline pt7 & $0.810(0.042)$ & $<0.001$ & $0.728,0.891$ & 0.655 \\
\hline pt8 & $0.844(0.040)$ & $<0.001$ & $0.766,0.922$ & 0.712 \\
\hline \multicolumn{5}{|c|}{ Memory related problems } \\
\hline RMBP1 & $0.745(0.063)$ & $<0.001$ & $0.621,0.868$ & 0.555 \\
\hline RMBP2 & $0.898(0.038)$ & $<0.001$ & $0.824,0.972$ & 0.806 \\
\hline RMBP3 & $0.837(0.041)$ & $<0.001$ & $0.756,0.917$ & 0.700 \\
\hline RMBP4 & $0.836(0.047)$ & $<0.001$ & $0.744,0.928$ & 0.699 \\
\hline RMBP5 & $0.837(0.045)$ & $<0.001$ & $0.748,0.926$ & 0.701 \\
\hline RMBP6 & $0.841(0.049)$ & $<0.001$ & $0.746,0.937$ & 0.708 \\
\hline RMBP7 & $0.777(0.059)$ & $<0.001$ & $0.661,0.893$ & 0.605 \\
\hline
\end{tabular}




\begin{tabular}{|l|l|l|l|l|l|}
\hline Depression & \multicolumn{1}{l|}{} & & \\
\hline RMBP12 & $0.644(0.068)$ & $<0.001$ & $0.512,0.777$ & 0.415 \\
\hline RMBP14 & $0.725(0.077)$ & $<0.001$ & $0.574,0.876$ & 0.526 \\
\hline RMBP17 & $0.790(0.046)$ & $<0.001$ & $0.700,0.879$ & 0.624 \\
\hline RMBP18 & $0.890(0.033)$ & $<0.001$ & $0.825,0.955$ & 0.793 \\
\hline RMBP19 & $0.788(0.046)$ & $<0.001$ & $0.697,0.878$ & 0.620 \\
\hline RMBP20 & $0.798(0.057)$ & $<0.001$ & $0.686,0.911$ & 0.638 \\
\hline RMBP21 & $0.848(0.041)$ & $<0.001$ & $0.768,0.928$ & 0.720 \\
\hline RMBP22 & $0.876(0.030)$ & $<0.001$ & $0.818,0.935$ & 0.768 \\
\hline RMBP23 & $0.816(0.043)$ & $<0.001$ & $0.732,0.899$ & 0.665 \\
\hline Disruption & & & & 0.438 \\
\hline RMBP8 & $0.661(0.081)$ & $<0.001$ & $0.502,0.821$ & 0.514 \\
\hline RMBP9 & $0.717(0.062)$ & $<0.001$ & $0.595,0.838$ & 0.248 \\
\hline RMBP10 & $0.498(0.090)$ & $<0.001$ & $0.321,0.675$ & 0.349 \\
\hline RMBP11 & $0.591(0.081)$ & $<0.001$ & $0.431,0.750$ & 0.439 \\
\hline RMBP13 & $0.662(0.085)$ & $<0.001$ & $0.496,0.828$ & 0.669 \\
\hline RMBP15 & $0.818(0.076)$ & $<0.001$ & $0.668,0.968$ & 0.669 \\
\hline RMBP16 & $0.818(0.054)$ & $<0.001$ & $0.711,0.924$ & 0.493 \\
\hline RMBP24 & $0.702(0.069)$ & $<0.001$ & $0.566,0.838$ & \\
\hline
\end{tabular}

\#Items.

on MR, $\alpha$, and $\omega$ every factor presents high reliability. The reported $\alpha$ of the three subscales namely; memory related problems, depression, and disruption were $0.93,0.93,0.87$ respectively. MR were: $0.95,0.97$, and 0.88 respectively. Hierarchical $\omega$ were: $0.94,0.94,0.86$ respectively. In addition, the AVE ranges from 0.47 to 0.68 representing overall high factor reliability and high item variance extracted.

Table 2 presents the factor loadings and $R^{2}$ for each factor. We reject the null hypothesis for every items factor loading $(p<0.001)$. Factor loadings range from 0.373 to 0.898 , with respective $R^{2}$ ranging from 0.139 to 0.806 . This shows that the factors explained between $13.9 \%$ and $80.6 \%$ of the variance in the items, the mean $R^{2}$ is $0.56(S D=0.18$, median $=0.61)$, in average across all items the factors explained $56 \%$ of the item variance.

For Embarrassment, the factor loadings ranged from 0.373 to 0.779 , and the $\mathrm{R}^{2}$ ranged from 0.139 to 0.607 , and in average 0.371 , indicating that the items in the factor shared in average $37.1 \%$ of their variance with the other items in the factor. In the case of patient dependency, the factor loadings ranged from 0.533 to 0.890 , and the respective $R^{2}$ ranged from 0.284 to 0.793 , indicating that the items in the factor shared in average $37.1 \%$ of their variance with the other items in the factor. While the factor loadings for self-criticism are set to be equal between the two items to 0.894 , with equal $R^{2}$ of 0.800 , meaning that the two items shared $80 \%$ of their variance that represents self-criticism.

For positive Thinking, the factor loadings ranged from 0.574 to 0.883 , with the respective $R^{2}$ of 0.329 to 0.779 , indicating that in average items shared $60.3 \%$ of their variance with other items in the factor. In the memory related problem factor, the factor loadings ranged from 0.745 to 0.898 , and the respective $R^{2}$ were 0.555 and 0.806 , showing that in average items shared $68.2 \%$ of their variance with the other items from the factor. In the case of the depression factor, the factor loadings ranged from 0.644 to 0.890 , with the respective $R^{2}$ of 0.415 and 0.793 , with an average of $64.1 \%$ of their variance shared with other depression items. Finally, for the disruption factor, the factor loadings ranged from 0.498 to 0.818 , with the respective $R^{2}$ of 0.248 and 0.669 , with an average of $47.7 \%$ of item variance shared with the other disruption items.

It is of interest to identify how informative are the items at each level of the construct. The parameters from the factor model (factor loadings and item thresholds) are transformed to calculate the Test Information Curves (TIC), which detail the amount of information that the items provide at different levels of the construct. Figure 1 presents the TIC for each construct. For Embarrassment, the items provide information at a large range of the construct, from low (2 SD below the mean) to high levels (4 SD above the mean). While for Reaction to patient dependency, and Self-Criticism the items still provide information for wide range of constructs, between -2 and +2 SD from the mean. The items for Positive Thinking and Memory related problems provided most of their information at low levels of the factors, below the mean. On the other direction, Depression and Disruption provide most of their information on higher levels of the constructs, above the mean. This means that Embarrassment, Reaction to patient dependency, and Self-Criticism are constructs that can describe subjects with low, medium, or high levels. On the other hand, Positive Thinking, and Memory related problems are more precise for subjects with medium and low levels of the construct. Finally, Depression and Disruption present precise information at medium and high levels of the constructs.

Lastly, Table 3 shows the factor correlations. The burden factors present positive correlations between each other $(p<0.05)$. Meaning that higher caregivers' 

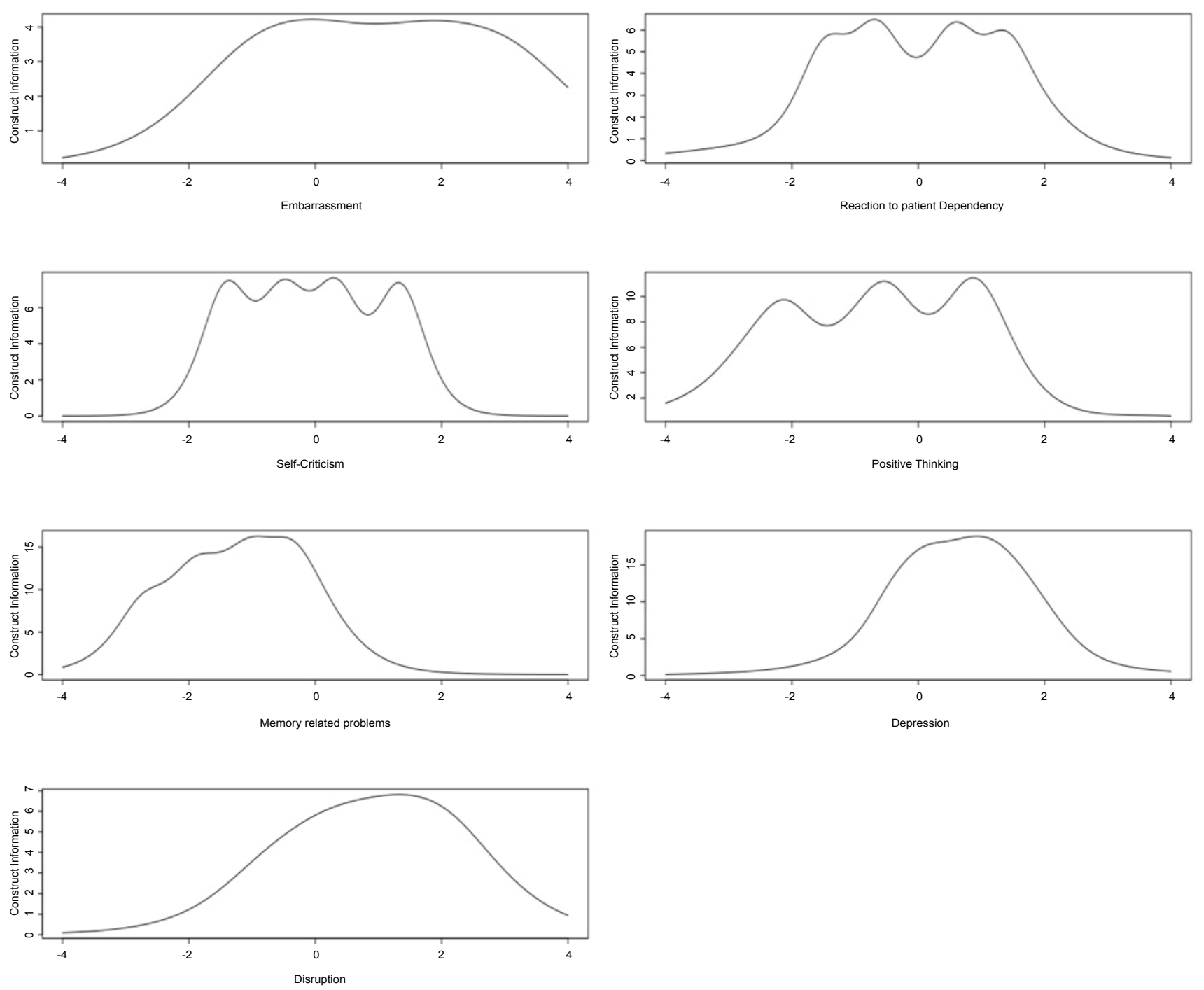

Figure 1: Test information curve for each underlying factor.

Table 3: Correlations between the underlying factors.

\begin{tabular}{|c|c|c|c|c|c|c|c|}
\hline & $\mathrm{Em}^{+}$ & $\mathbf{P d p}^{++}$ & SC $^{\wedge}$ & $P T^{\wedge \wedge}$ & Mem $^{* *}$ & $\operatorname{Dep}^{* * *}$ & Dis $^{+++}$ \\
\hline $\mathrm{Em}^{+}$ & 1 & & & & & & \\
\hline $\mathrm{Pdp}^{++}$ & $0.890^{*}$ & 1 & & & & & \\
\hline$S^{\wedge}$ & $0.490^{*}$ & $0.434^{*}$ & 1 & & & & \\
\hline $\mathrm{PT}^{\wedge \wedge}$ & $-0.393^{*}$ & $-0.395^{*}$ & $-0.226^{*}$ & 1 & & & \\
\hline $\mathrm{Mem}^{* *}$ & 0.173 & $0.231^{*}$ & 0.114 & -0.010 & 1 & & \\
\hline $\operatorname{Dep}^{* \star *}$ & 0.059 & -0.055 & 0.111 & 0.006 & 0.171 & 1 & \\
\hline $\mathrm{Dis}^{+++}$ & $0.398^{*}$ & $0.330^{*}$ & 0.143 & -0.109 & $0.354^{*}$ & $0.660^{*}$ & 1 \\
\hline
\end{tabular}

" $\mathrm{p}<0.05$; ${ }^{+}$Embarrassment; ${ }^{++}$Reaction to patient dependency; ' Self-criticism; ${ }^{\wedge}$ Positive thinking; ${ }^{* *}$ Memory related problems; ***Depression; ${ }^{+++}$Disruption.

embarrassment/anger is associated with higher reaction to patient's dependency and to self-criticism, and higher Patient's dependency is associated with higher self-criticism. Positive thinking has negative correlations with the burden factors. Meaning that higher caregivers' positive thinking is associated with lower caregivers' embarrassment and lower reaction to patient dependency, and self-criticism, while its correlations with memory, disruption, and depression are not different from $0(p>0.05)$. Memory and depression have few correlations different from $0(p<$ $0.05)$, both have a positive correlation with disruption. In other words, higher patient's memory related problems and depression are associated with higher patient's disruption. Also, patient memory related problems have positive correlation to caregivers' reaction to patient's dependency. Finally, patient's disruption has a positive correlation with caregivers' embarrassment and to caregivers' reaction to patient's dependency $(p<0.05)$.

These correlations are also testing the construct validity of the scales. As the correlation can be match to theoretical relation between them. As the construct relations are theoretically sounds, we can determine that the factors are precise (reliability) measures of 
theoretically constructs that are intending to measure $[22,28,29,46]$.

\section{Discussion}

To date, this is the first study that investigated the factorial structure of three theoretically related scales, namely, the PTSS, RMBPC, and ZBI, among caregivers of persons with dementia. In addition, the study is the first that investigated the reliability of each factor within these three scales and their correlation with each other. The results of the present study support the use of the three subscales of the Revised Memory and Behavior Problems Checklist (RMBPC) as a measure of behavioral problems in patients with dementia. This analysis confirmed three factors namely, memory-related problems, depression, and disruption, indicating that the three-factorial structure is a good representation of the data. The RMBPC has clinical implications. First, it can be used as a screening measure to identify specific behavior problems in persons with dementia. Second, the measure can be administered over time to identify the disease progression as well as to evaluate the treatment/intervention given and its impact on patients' behaviors. Finally, the three subscales can provide more specific assessment for the patient's behaviors so that adequate interventions can be tailored to patients' needs.

To date, this is the first study that evaluated the reliability of the RMBPC using multiple indices. This study uses the most common estimate of reliability which is the Cronbach's coefficient alpha $(\alpha, 1951)$ [40] as well as two improved estimates of reliability; hierarchical $\omega$ [42] and the Maximal Reliability (MR) coefficient. In addition, these estimates of construct reliability are presented with their respective Average Variance Extracted (AVE) [44]. The use of multiple (improved) measures of reliability leads to a more accurate measure of these characteristics. For future research, these are better representation of the expected reliability for the underlying factors. Hierarchical $\omega$ is a conservative measure of reliability, while MR measures the highest expected reliability. Between them, we can look at the possible range for factor reliability, which presented as high for all factors. Meaning that the shared variance between items related to the underlying factors is high across factors.

The results of the present study support the use of the three subscales of the ZBI namely; embarrassment/ anger, reaction to patients' dependency, and selfcriticism as a measurement of caregiver burden. Isik and colleagues [47], pointed out the fact that caregivers of persons with dementia are experienced more burden and anxiety as compared to caregivers of older adults because they have to cope with both agerelated conditions and dementia related factors, which could worsen the relationship between caregivers and patients [47]. Also, Chiao and colleagues [48] in their systematic review of dementia caregivers' burden pointed out the fact that many studies are using the ZBI, specifically among the 21 studies that they reviewed, 10 studies used the English version of the $\mathrm{ZBI}$ and two studies used the Spanish version of the ZBI [48]. Therefore, the three subscales of the ZBI can help health care professionals to target specific areas of assessment for caregivers so that adequate interventions can be tailored to patients' needs. The scale has implications for practice. For example, screening caregivers, especially those who received a recent diagnosis for their beloved one, is essential to decrease the possibility of developing depression. Recognizing their burden early can help health care professionals to direct their primary prevention by promoting caregivers' wellness by preventing or decreasing the stress. Again, this is the first study that evaluated the reliability of the $\mathrm{ZBI}$ using multiple indices and the results indicated that $\mathrm{ZBI}$ is a reliable measure for caregivers' burden assessment. Similarly, the PTSS was designed to be used in empirical and clinical studies. Specifically, assessing the eight skills constituting the PTSS can help health care professionals to identify which skills are used by caregivers so, that they can be strengthened, and which are not, so those skills can be taught.

Regarding the factor correlations of the scales, as expected, the burden factors present positive correlations between each other. Positive thinking has negative correlations with the burden factors. Memory related problems and depression have a positive correlation with disruption. Also, patient memory related problems have positive correlation to caregivers' reaction to patient's dependency. Finally, patient's disruption has a positive correlation with caregivers' embarrassment and to caregivers' reaction to patient's dependency.

In sum, the results of this study indicated that the factorial structure of the three scales is a good representation of the data. Also, the results of the study indicated an acceptable reliability of each factor of the three measures. Finally, the factor correlated as expected and showed its relevance to the underlying theory. These correlations represent construct validity, as the represented constructs followed the expected theoretical relations. Future studies might consider studying the mediating and the moderating effects of positive thinking on care-recipients challenging behavior problems. In fact, the systematic review conducted by $\mathrm{Wu}$ and colleagues [49], showed that most of the interventions showed a potential to improve the health and well-being of dementia caregivers and care recipients. In addition, the study conducted by Hughes and colleagues [50], concluded that addressing the potentially modifiable unmet caregiver needs can reduce caregiver burden. Therefore, the findings from this study along with the findings from the future mediator/moderator study, can be used as a guide to 
provide a positive thinking training intervention among caregivers of persons with dementia to help decrease their burden, which eventually will impact their care recipients challenging behavior.

\section{References}

1. Alzheimer's Association (2012) 2012 Alzheimer 's disease facts and figures. Alzheimers Dement 8: 131-168.

2. Bekhet A, Zauszniewski JA (2013) Psychometric assessment of the depressive cognition scale in caregivers of persons with dementia. Issues in Mental Health Nursing 34: 678-684.

3. Papastavrou E, Kalokerinou A, Papacostas SS, Tsangari $H$, Sourtzi $P$ (2007) Caring for a relative with dementia: Family caregiver burden. J Adv Nurs 58: 446-457.

4. Bekhet A (2013) Effects of positive cognitions and resourcefulness on caregiver burden among caregivers of persons with dementia. Int J Ment Health Nurs 22: 340-346.

5. Coon DW, Rubert M, Solano N, Mausbach B, Kraemer H, et al. (2004) Well-being, appraisal, and coping in latina and caucasian female dementia caregivers: Findings from the REACH study. Aging Ment Health 8: 330-345.

6. Chenoweth B, Spencer B (1986) Dementia: The experience of family caregivers. Gerontologist 26: 267-272.

7. Loos C, Bowd A (1997) Caregivers of persons with alzheimer's disease: Some neglected implications of the experience of personal loss and grief. Death Stud 21: 501514.

8. Parsons K (1997) The male experience of caregiving for a family member with alzheimer's disease. Qualitative Health Research 7: 391-407.

9. Bekhet A, Sjostedt J (2018) Resilience in the lives of caregivers of persons with dementia: Caregivers' Perspectives. Archives of Psychiatric Nursing 32: 19-23.

10. Cuijpers $P$ (2005) Depressive disorders in caregivers of dementia patients: A systematic review. Aging Ment Health 9: 325-330.

11. Joling KJ, Van Hout HPJ, Schellevis FG, Van der Horst HE, Scheltens P, et al. (2010) Incidence of depression and anxiety in the spouses of patients with dementia: A naturalistic cohort study of recorded morbidity with a 6-year follow-up. The American Journal of Geriatric Psychiatry 18: 146-153.

12. Depp C, Sorocco K, Kast-Godley J, Thompson L, Rabinowitz $\mathrm{Y}$, et al. (2005) Caregiver self-efficacy, ethnicity, and kinship differences in dementia caregivers. Am J Geriatr Psychiatry 13: 787-794.

13. Duggleby W, Williams A, Wright K, Bollinger S (2009) Renewing everyday hope: The hope experience of family caregivers of persons with dementia. Issues in Mental Health Nursing 30: 514-521.

14. Roth DL, Burgio LD, Gitlin LN, Gallagher-Thompson D, Coon DW, et al. (2003) Psychometric analysis of the revised memory and behavior problems checklist: Factor structure of occurrence and reaction ratings. Psychol Aging 18: $906-915$

15. Teri L, Truax P, Logsdon R, Uomoto J, Zarit S, et al. (1992) Assessment of behavioral problems in dementia: The revised memory and behavior problems checklist. Psychol Aging 7: 622-631.

16. Dura JR, Bornstein RA, Kiecolt-Glaser JK (1990) Refinements in the assessment of dementia-related behaviors: Factor structure of the memory and behavior problem checklist. Psychological Assessment 2: 129-133.

17. Fuh JL, Liu CY, Wang SJ, Wang HC, Liu HC (1999) Revised memory and behavior problems checklist in Taiwanese patients with alzheimer's disease. Int Psychogeriatr 11: 181-189.

18. Park HS, Dailey R, Lemus D (2002) The use of exploratory factor analysis and principal components analysis in communication research. Human Communication Research 28: 562-577.

19. Preacher KJ, MacCallum RC (2003) Repairing tom swift's electric factor analysis machine. Understanding Statistics 2: $13-43$.

20. Lai DW (2007) Validation of the zarit burden interview for Chinese Canadian caregivers. National Association of Social workers 31: 45-53.

21. Hebert R, Bravo G, Preville M (2000) Reliability, validity and reference values of the zarit burden interview for assessing informal caregivers of community-dwelling older persons with dementia. Canadian Journal on Aging 19: 494-507.

22. Knight BG, Fox LS, Chou C (2000) Factor structure of the burden interview. Journal of Clinical Geropsychology 6: 249-258.

23. Rankin ED, Haut MW, Keefover RW, Franzen MD (1994) The establishment of clinical cutoffs in measuring caregiver burden in dementia. Gerontologist 34: 828-832.

24. Whitlatch CJ, Zarit SH, von Eye A (1991) Efficacy of interventions with caregivers: A reanalysis. Gerontologist 31: 9-14.

25. Longmire CF, Knight BG (2011) Confirmatory factor analysis of a brief version of the zarit burden interview in black and white dementia caregivers. Gerontologist 51: 453-462.

26. Gallagher D, Lovett S, Zeiss A (1985) Interventions with caregivers of frail elderly persons. In: Ory M, Bond K, Aging and Health Care: Social Science and Policy Perspectives. Tavistock, New York, USA, 167-190.

27. Thompson EH, Futterman AM, Gallagher-Thompson D, Rose JM, Lovett SB (1993) Social support and caregiving burden in family caregivers of frail elders. J Gerontol 48: S245-S254.

28. Bekhet A, Zauszniewski J (2013) Measuring use of positive thinking skills scale: Psychometric testing of a new scale. West J Nurs Res 35: 1074-1093.

29. Liu Y, Insel KC, Reed PG, Crist JD (2012) Family caregiving of older Chinese people with dementia: Testing a model. Nurs Res 61: 39-50.

30. https://www.R-project.org/

31. Rosseel Y (2012) Lavaan: An R package for structural equation modeling. Journal of Statistical Software 48: 1-36.

32. Kline RB (2016) Principles and practice of structural equation modeling. ( $4^{\text {th }}$ edn), Guilford Press, New York, USA.

33. Little TD (2013) Longitudinal structural equation modeling. Methodology in the social sciences. The Guilfors Press, New York, USA.

34. Raykov T (2012) Scale construction and development using structural equation modeling. In: Hoyle RH, Handbook of structural equation modeling. Guilford, New York, USA, 472-494. 
35. Baraldi AN, Enders CK (2010) An introduction to modern missing data analyses. J Sch Psychol 48: 5-37.

36. Enders CK (2010) Applied missing data analysis. Guilford Press, New York, USA.

37. Van Buuren S (2012) Flexible imputation of missing data. Chapman \& Hall/CRC.

38. Van Buuren S, Groothuis-Oudshoorn K (2011) Mice: Multivariate imputation by chained equations. Journal of Statistical Software 45: 1-67.

39. semTools Contributors (2018) semTools: Useful tools for structural equation modeling.

40. Cronbach LJ (1951) Coefficient alpha and the internal structure of tests. Psychometrika 16: 297-334.

41. Brown TA (2015) Confirmatory factor analysis for applied research. ( $2^{\text {nd }}$ edn), Guilford Press, New York, USA.

42. McDonald RP (1999) Test theory: A unified treatment. Lawrence Erlbaum Associates Publishers, NJ, USA.

43. Li H (1997) A unifying expression for the maximal reliability of a linear composite. Psychometrika 62: 245-249.

44. Fornell C, Larcker DF (1981) Evaluating structural equation models with unobservable variables and measurement errors. Journal of Marketing Research 18: 39-50.
45. Bovaird JA, Kozoil NA (2012) Measurement models for ordered-categorical indicators. In: Hoyle RH, Handbook of structural equation modeling. Guilford, New York, USA, 495-511.

46. DeVon HA, Block ME, Moyle-Wright $P$, Ernst DM, Hayden SJ, et al. (2007) A psychometric toolbox for testing validity and reliability. J Nurs Scholarsh 39: 155-164.

47. Isik AT, Soysal P, Solim M, Veronese N (2018) Bidirectional relationship between caregiver burden and neuropsychiatric symptoms in patients with Alzheimer's Disease: A narrative review. International Journal of Geriatric Psychiatry.

48. Chiao CY, Wu HS, Hsiao CY (2015) Caregiver burden for informal caregivers of patients with dementia: A systematic review. Int Nurs Rev 62: 340-350.

49. Wu B, Petrovsky DV, Wang J, Xu H, Zhu Z, et al. (2018) Dementia caregiver interventions in chinese population: $A$ systematic review. J Adv Nurs.

50. Hughes TB, Black BS, Albert M, Gitlin LN, Johnson DM, et al. (2014) Correlates of objective and subjective measures of caregiver burden among dementia caregivers: Influence of unmet patient and caregiver dementia-related care needs. Int Psychogeriatr 26: 1875-1883. 